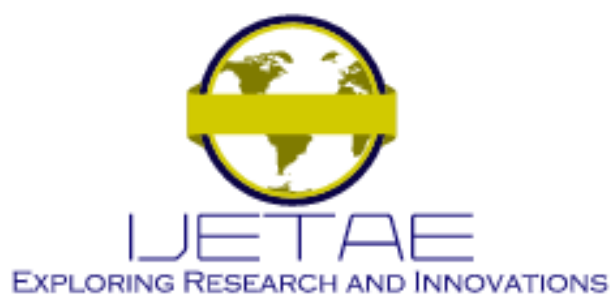

International Journal of Emerging Technology and Advanced Engineering

Website: www.ijetae.com (E-ISSN 2250-2459, Scopus Indexed, ISO 9001:2008 Certified Journal, Volume 12, Issue 01, January 2022)

Manuscript Received: 12 October 2021, Received in Revised form: 03 January 2022, Accepted: 10 January 2022

DOI: 10.46338/ijetae0122_13

\title{
Bare Skin Image Classification using Convolution Neural Network
}

\author{
Jaya Gupta ${ }^{1}$, Sunil Pathak ${ }^{2}$, Gireesh Kumar ${ }^{3}$ \\ ${ }^{1,2}$ Amity School of Engineering and Technology, Department of Computer Science \& Engineering, Amity University Rajasthan, \\ Jaipur, India. \\ ${ }^{3}$ Institute of Engineering \& Technology, Department of Computer Science \& Engineering, JK Lakshmipat University Jaipur,
} Rajasthan, India.

\begin{abstract}
Image classification is critical and significant research problems in computer vision applications such as facial expression classification, satellite image classification, and plant classification based on images. Here in the paper, the image classification model is applied for identifying the display of daunting pictures on the internet. The proposed model uses Convolution neural network to identify these images and filter them through different blocks of the network, so that it can be classified accurately. The model will work as an extension to the web browser and will work on all websites when activated. The extension will be blurring the images and deactivating the links on web pages. This means that it will scan the entire web page and find all the daunting images present on that page. Then we will blur those images before they are loaded and the children could see them.
\end{abstract}

Keywords- Activation Function, CNN, Images Classification, Optimizers, VGG-19.

\section{INTRODUCTION}

As we are living in the world of the Internet, Social Media and Browsing has become an important part of our life. Not only adults but even kids spend most of their time on the mobiles browsing some information on different websites or on social media. As the use of Internet is growing tremendously, it has also become a very important medium for the advertisements of the product. Traditionally, the banners or pamphlets were used for the promotion of any item. Now a days, the advertisements are just displayed on the social media like Facebook, Instagram or on the most visited websites on the internet. It may happen that while browsing, children may come across some web pages on the internet which may be exposed or nude image of a human. It is not good for the children to see such type of images in such an early age where their mind is in developing phase and they don't even know about this.
For this parental controls are available, but in this we can restrict the children to some limited sites. So we need a user friendly application which is easy to use and free of cost. The proposal will create a chrome extension which just needs to be downloaded and it will start its work without any prior setup in your personal computer.

Convolution Neural Network (CNN) is one of the most popular and effective technology for image recognition and image classification. The CNN classifier takes the image as an input, processes it and classifies it under certain categories .As CNN takes image as an input, we need a good dataset on which CNN model will be trained. Convolution neural networks are used widely for image classification applications .In the literature it has been used for Traffic Scene Classification on a Representation Budget by S.Ivan et al. [1], for wide band remote sensingimage classification by Xinyu Lei et al.[2] ,for skin disease classification by Parameshwar R. Hegde et al. [4],for Detection and Classification of Lung Abnormalities by Shoji Kido et al.[5],for remote sensing image classification by $\mathrm{Na}$ Liu et al. [6] , Pulmonary Artery-Vein Classification by Pietro Nardelli et al. [7], Classification of Fine-grained Fashion Image by Yian Seo et al. [8], for Classifying Body Constitution Based on Face Image by Er-Yang Huan et al. [9],for Advertisement Image Classification by An Tien Vo et al. [12],for classification of medical images by [14][17][18]. The proposed model uses Convolutional Neural Network (CNN) to identify these images and filter them through different blocks of the network, so that it can be classified accurately. The model works on TensorFlow, a user friendly platform, which provides different high leveled APIs (in Keras) which are used to build any basic model. It also permits us to add our own libraries. The input data for the model are images we collected online through various re-sources. 


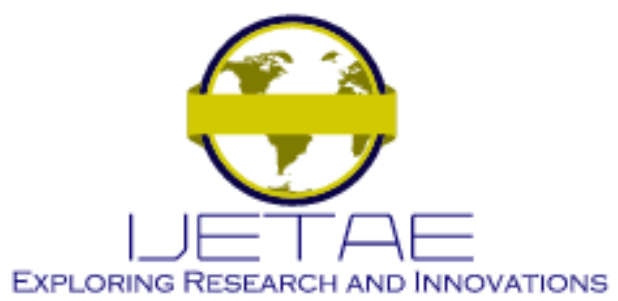

\section{International Journal of Emerging Technology and Advanced Engineering}

Website: www.ijetae.com (E-ISSN 2250-2459, Scopus Indexed, ISO 9001:2008 Certified Journal, Volume 12, Issue 01, January 2022)

\section{LITERATURE REVIEW}

The Convolutional Neural Network (CNN) classifies the image regions as a collection of either skin or non-skin regions, it also classifies it based on the curves and edges it encounters in the image. Various approaches to skin modelling are used in the literature. Here we give a brief review of the neural net-work models for skin detection. One approach is a learning scheme based on the skin colour distribution of the image, using a neural network to learn and classify whether the input image contains skin exposure.

Earlier work on nude identification focused on human skin detection, in which the idea is that greater amounts of detected skin would lead to higher probabilities of nudity within the image, hence characterizing the content as nude. Nevertheless, these approaches suffer with a high rate of false positives, especially in the context of beaches or practice of aquatic sports. An Tien Vo et al. suggested a method which involved ConvNetJS an open library which implements Deep Convolutional Neural Network[9]. It is written in JavaScript and HTML with various supporting features for research purpose. It is an effective Neural Network, and is able to use CPU instead of GPU [11]. The model proposed by them is an advertisement image classification system based on $\mathrm{CNN}$ consisting of 4 steps. It takes the image as an input from the webpage. It takes the image on the web browser by skin capturing and then saving that image and then at the end classifying it by passing it through the CNN model. Our model is based on real time image disabling, so using this method would not work in our case.

A residual learning framework to ease the training of networks that are substantially deeper than those used previously was put forth by [15][20-26]. They explicitly reformulated the layers as learning residual functions with reference to the layer in-puts, instead of learning unreferenced functions. They provided comprehensive empirical evidence showing that these residual networks are easier to optimize, and can gain accuracy from considerably increased depth. Another approach is to use dilated CNN to reduce the complexity and improve the classification accuracy by [3][10].

Apart from this the model proposed by [35] contained two types of skin models used in the literature viz., symmetric and asymmetric.
Symmetric model uses a single classifier for both the classes whereas asymmetric model uses two separate classifiers for skin and non-skin pixels that are separately trained using respective features. Advantage of asymmetric skin classifier is that it increases the distances in certain skin related features between a positive (skin) and a negative (non-skin) image, with disadvantage of increased time complexity for training two classifiers. The neural classifiers used in the literature either uses a symmetric model with single neuron in the output layer or uses two separate neural networks (asymmetric model) for each of the skin and non-skin classes. The novelty of our approach is that it has multiple convolutional that is hidden layer working on only skin colour images containing humans. Other image classification approaches based on zero shot and multi stage features are also given by [16][19].

In [27] Convolutional Neural Network using deep learning was used for detection and classification of objects related to metal polishing shaft surface. In [28], analysis were carried out to periodicity and saliency for adult video detection. Pornographic images and bikini images were classified using machine learning based forensic discriminator [29]. In [30], a new algorithm was pro-posed which is known as Coyote optimization algorithm using metaheuristic for global optimization problems. SHyAg hunters based on the characteristics of Aquila hunter and the hyena hunters are hybridized based on [31] using SHyAq optimization algorithms, which compare the features of quila Hunters and the hyena hunters. In [32], propose texture description for texture classification using local directional ternary pattern. In [33], feature selection was carried out using binary spotted hyena optimizer for finding out various selected feature from image. Aquila Optimizer [34] using novel meta heuristic optimization algorithms was proposed for identifying various characteristics from given input image.

\section{PROPOSED WORK AND IMPLEMENTATION}

All paragraphs must be indented. All paragraphs must be justified, i.e. both left-justified and right-justified.

\section{A. Dataset}

First objective was the collection of a good dataset which is the most important part while working in CNN. 


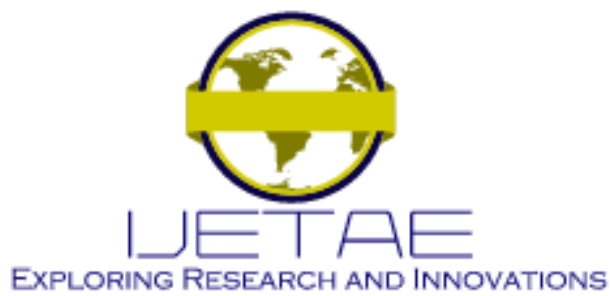

International Journal of Emerging Technology and Advanced Engineering Website: www.ijetae.com (E-ISSN 2250-2459, Scopus Indexed, ISO 9001:2008 Certified Journal, Volume 12, Issue 01, January 2022)

As this is a very sensitive thing, the datasets are not readily available on the internet, hence we need to scrap images from the internet. The .txt files contain the links of both nude and non-nude images which are used for creation of dataset. Python library named "urllib.request" is used for scraping the images from the internet. Syntax for the same is "urllib.request.urlretrieve(url,name)", We need to pass 2 parameters, first is the variable url which has links and variable name which contains the string in which we want the image to be saved. The images are saved in the same directory where the code is present. Currently the dataset which is used for training the model has 5600 training images and 1000 testing images.

\section{B. State of the Art CNN Models}

Once the dataset is collected, next step is working on CNN model for training. The image size needs to be fixed before passing it from the model which is kept (200,200).There is many pre-defined CNN models available which are listed below [13]:

- LeNet-5

- AlexNet

- VGG16 and VGG19

- Inception-v1 and Inception-v3 and Inception-v4

- ResNet-50

- Xception

All this models have different use in itself and also with different number of layers. But for our dataset we used VGG19, which has 19 layers out of which 16 are convLayers and 3 are fully connected layers, last 3 fully connected layers are removed and manually 4 fully connected layers are added with filter value 500.With this model, the accuracy on 15 epochs is $85.39 \%$.

\section{Proposed CNN Architecture}

Above was the work done with pre-defined VGG19 model. Creation of the own model is also done where the no. of layers, value of filters, values of kernels, no. of fully connected layers all are chosen by trial and error. Figure 1 shows the working or Flow of Convolution Neural Network.

The images are first need to be pre-processed before passing from the model. As the computer do not understand image in the image format, it understands this in the form of pixel values. Hence we need to store pixel value of image in list and we need to convert them into categorical data as well in the other list i.e. 0 for non-nude and 1 for nude. This process is done both on training and testing dataset. Then this list is passed through model for training and finally prediction is done.

Few parameters are kept constant for both the models:

Optimizer Used : Stochastic Gradient Descent(SGD)

Activation Function : Sigmoid

Learning Rate : 0.001

Momentum : 0.9

Batch Size : 128

Loss : Binary Crossentropy

No. of Epochs : 15

If the images are nude, the model will predict output as 1 else 0 .As the accuracy is between 85 to 88 ,there is a possibility of predicting the values in decimal like 0.000045 ,for this a threshold is kept if value if $>0.5$ then nude else non-nude. 


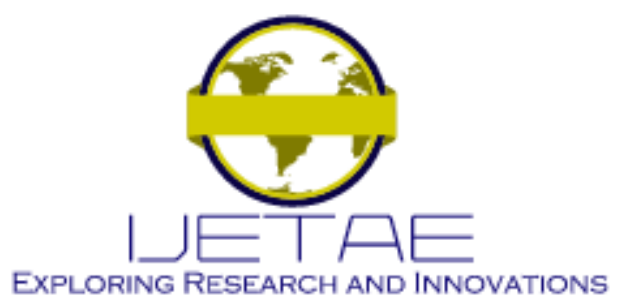

International Journal of Emerging Technology and Advanced Engineering

Website: www.ijetae.com (E-ISSN 2250-2459, Scopus Indexed, ISO 9001:2008 Certified Journal, Volume 12, Issue 01, January 2022)

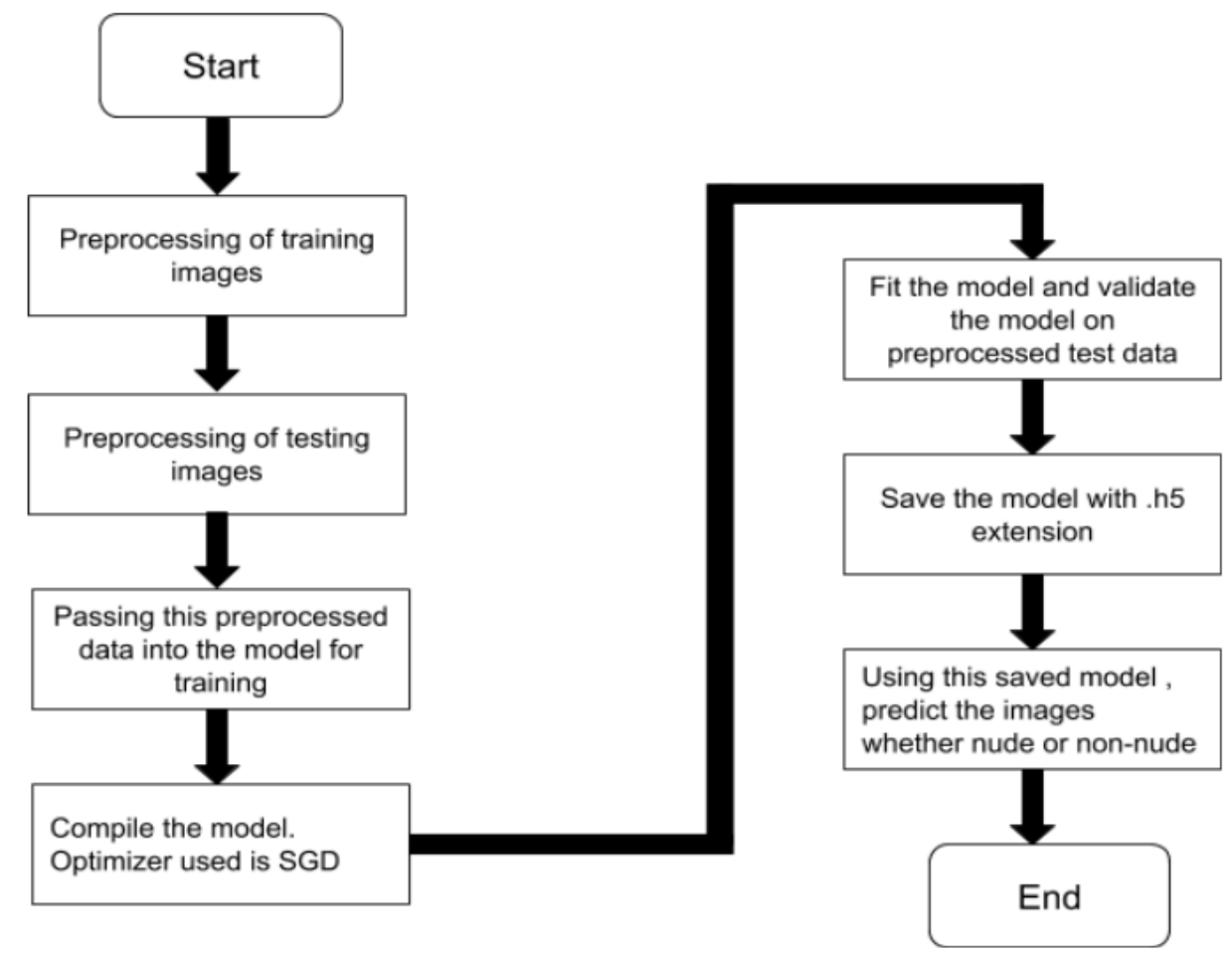

Fig. 1. Working or Flow of Convolution Neural Network(CNN)

Keras Sequential model allows us to add Convolution layers according to our choice. We can add as many number of convolution layers as we need according to the requirements. We need three basic components to define a basic convolutional network:

1. The Convolution Layer

2. The Pooling Layer

3. The Outer Layer

After this we can say that the model which was created on our own works best. Activation functions introduce nonlinear properties to Neural Network. Their main purpose is to convert an input signal of a node to an output signal. That output signal now is used as an input in the next layer in the stack. Specifically in an Artificial Neural Network we do the sum of products of in-puts(X) and their corresponding Weights (W) and apply an Activation function $f(x)$ to it to get the output of that layer and feed it as an input to the next layer.

Optimizers tie together the loss functions and model parameters by updating the model in response to the output of the loss.
$S G D$ :

SGD stands for Stochastic Gradient Descent. It is the most widely used and best optimizer while working with the images. The gradient descent algorithm batches the images for each iteration. Here batch considers all the images in the dataset which may create noise in the model. Hence, solution to this problem is SGD which has the batch size on one image and it processes only one image in iteration. Also SGD selects the image at random for processing.

\section{Sigmoid:}

The reason why sigmoid is considered to be the best activation function is that its value ranges from 0 to 1 .It works best when we want the prediction in the probability. As the proposed model has only 2 class nude and non-nude , probability will be 0 or 1 or value may lie in between them which varies de-pending on the image. 


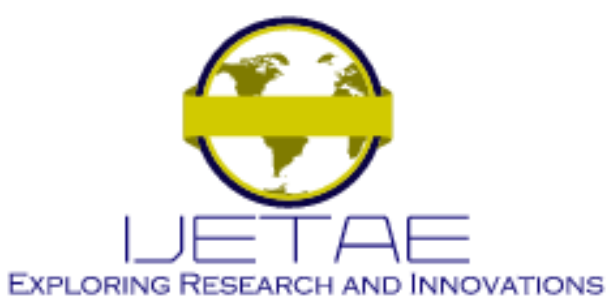

International Journal of Emerging Technology and Advanced Engineering

Website: www.ijetae.com (E-ISSN 2250-2459, Scopus Indexed, ISO 9001:2008 Certified Journal, Volume 12, Issue 01, January 2022)

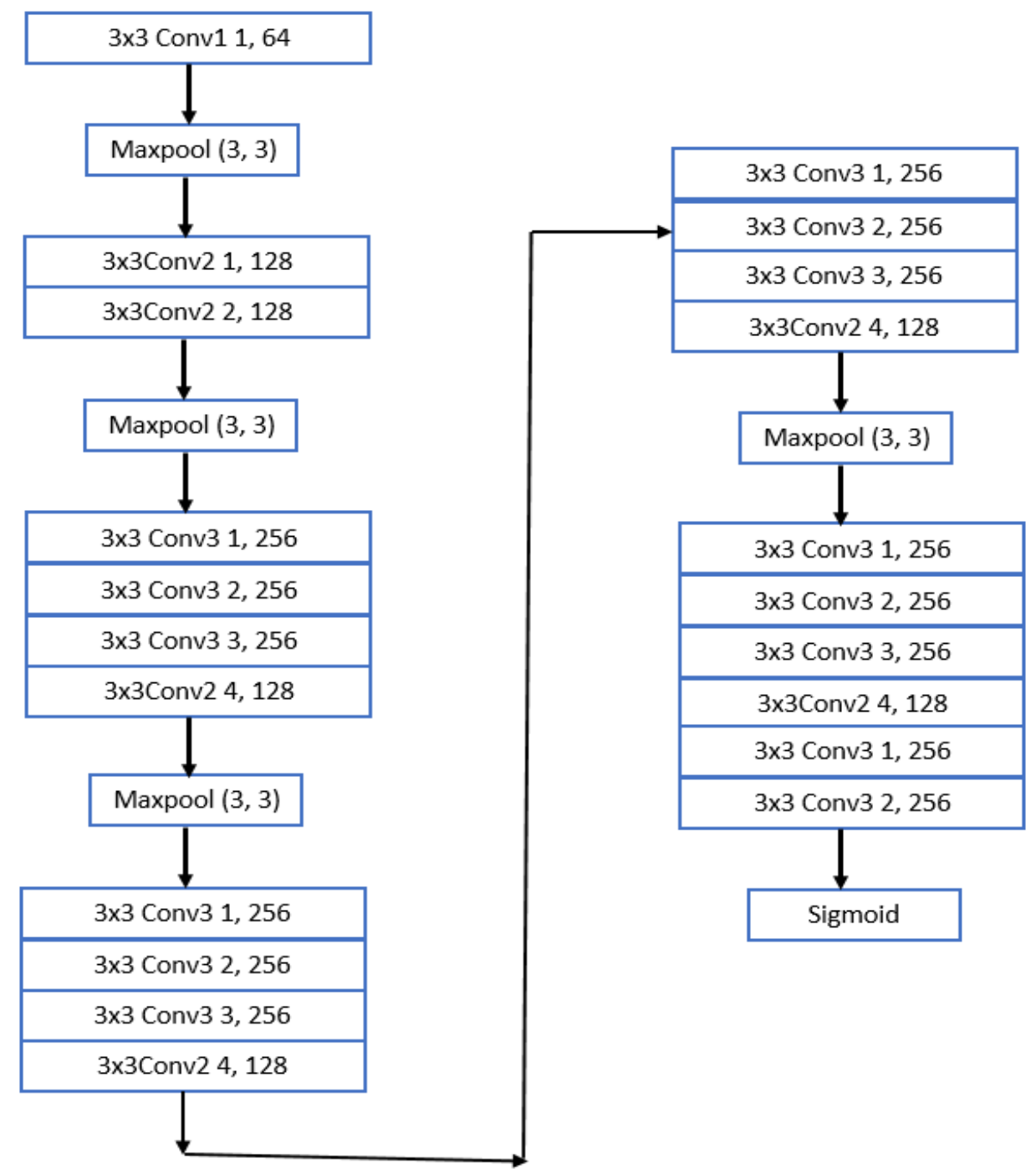

Fig. 2. Details of Convolution Layers of proposed model 


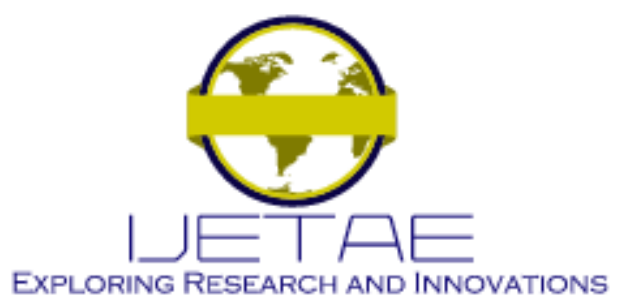

International Journal of Emerging Technology and Advanced Engineering

Website: www.ijetae.com (E-ISSN 2250-2459, Scopus Indexed, ISO 9001:2008 Certified Journal, Volume 12, Issue 01, January 2022)

Sometimes overfitting may occur in the model. Overfitting means the model will correctly predict only for the images which are used while training the model. So to check whether our model is overfitted or not, we need to pass the images which are not used while training the model. And the model created for this project is working good with the images other than used in training the model, hence there is no issue of overfitting in this case.

As we are completely ready with the working model with good accuracy, now it's time to use the saved model for creating the chrome extension. Chrome extension does not understand the .h5 extension files, we need to convert this model into the .json format. For the conversion of .h5 to .json, python package "tensorflowjs" is installed and command "tensor-flowjs_converter --input_format=keras /path_to_file/model.h5/path_to_file/tfjs_model". This json file will be used for model prediction.

This chrome extension first scraps the url of the images present in IMG tag then the images needs to be resized in 200X200 pixels as we have trained CNN model with 200X200 image size, then pass this image through .json file which will predict whether image is nude or non-nude. If nude, the chrome extension will blur the image and disable the link if applicable.

\section{RESUlt ANALysis}

We used 5600 training images and 1000 testing images for VGG-19 (16 convolution layers +3 fc layers) and proposed CNN architecture which is shown in figure 2(16 convolution layers $+7 \mathrm{fc}$ layers). SGD Optimizer and Sigmoid activation function are used for both. Table 1 shows the accuracy results of both the models for 10 and 15 epochs. As seen in Table 1, proposed Convolution Neural Network architecture is giving the accuracy of $87.29 \%$ for 15 epochs and performing better than VGG-19 for our dataset.

Python was used for implementation with below hardware specification.

- Processor - Intel Xeon E2630 v4 - 10 core processor, $2.2 \mathrm{GHz}$ with Turboboost upto $3.1 \mathrm{GHz}$.

- Motherboard - ASRock EPC612D8A.

- $\quad$ RAM - 128 GB DDR4 $2133 \mathrm{MHz}$.

- 2 TB Hard Disk (7200 RPM) + 512 GB SSD.

- $\quad$ GPU - NVidia TitanX Pascal (12 GB VRAM)
The following Table 1 shows the accuracy results of both the models for 10 and 15 epochs. As seen in Table 1, proposed Convolution Neural Network architecture is giving the accuracy of $87.29 \%$ for 15 epochs and performing better than VGG-19 for our dataset.

TABLE I

ACCURACY FOR VGG-19 AND PROPOSED CNN ARCHITECTURE FOR 10 AND 15 EPOCHS

\begin{tabular}{|l|c|c|}
\hline Model Name & No. of Epochs & Accuracy \% \\
\hline VGG-19 & 10 & 84.28 \\
\hline $\begin{array}{l}\text { Proposed CNN } \\
\text { Model }\end{array}$ & 10 & 86.39 \\
\hline VGG-19 & 15 & 85.39 \\
\hline $\begin{array}{l}\text { Proposed CNN } \\
\text { Model }\end{array}$ & 15 & 87.29 \\
\hline
\end{tabular}

\section{CONCLUSION AND FUtURE WORK}

A typical CNN often consists of several convolutional and fully-connected layers. The exact number of layers generally depends on the requirement of network capacity and memory cost for a specific classification task. The research work undertaken deals with classifying bare skin images and safe to work images using an existing CNN architecture model (VGG19) and a pro-posed CNN model and comparing accuracy of both the architectures .Here, we are getting $85.39 \%$ accuracy using VGG19 model and $87.29 \%$ accuracy for proposed CNN model for 15 epochs. In future we can work on animated images and videos to classify whether they are safe to work or not.

\section{REFERENCES}

[1] S. Ivan, B. Karla, and B. Peter, "Traffic Scene Classification on a Representation Budget", IEEE Transactions on Intelligent Transportation Systems, pp. 1-10, 2020.

[2] Xinyu Lei, Hongguang Pan_, Xiangdong Huang, “A dilated CNN model for image classification",IEEE Access vol. 7, DOI: 10.1109/ACCESS.2019.2927169 , pp. 124087 - 124095, July 2019.

[3] Shyava Tripathi, Rishi Kumar, "Image Classification using small Convolutional Neural Network", 2019 9th International Conference on Cloud Computing, Data Science \& Engineering (Confluence), DOI: $10.1109 /$ CONFLUENCE.2019.8776982,pp. 483-487 ,July 2019. 


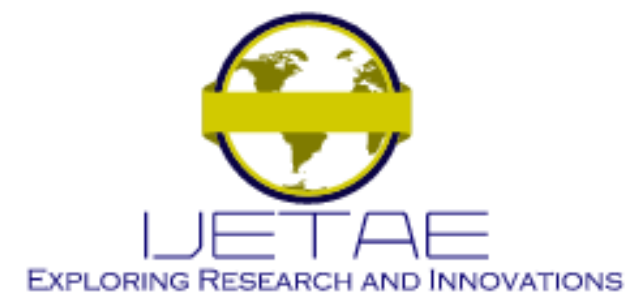

International Journal of Emerging Technology and Advanced Engineering

Website: www.ijetae.com (E-ISSN 2250-2459, Scopus Indexed, ISO 9001:2008 Certified Journal, Volume 12, Issue 01, January 2022)

[4] Parameshwar R. Hegde, Manjunath M. Shenoy , B.H. Shekar, "Comparison of Machine Learning Algorithms for Skin Disease Classification Using Color and Texture Features", 2018 International Conference on Advances in Computing, Communications and Informatics (ICACCI), DOI: 10.1109/ICACCI.2018.8554512, pp. 1825-1828,December 2018.

[5] Shoji Kido, Yasusi Hirano, Noriaki Hashimoto "Detection and Classification of Lung Abnormalities by Use of Convolutional Neural Network (CNN) and Regions with CNN Features (R-CNN)" ,2018 International Workshop on Advanced Image Technology (IWAIT), DOI: 10.1109/IWAIT.2018.8369798,May 2018.

[6] Na Liu, Lihong Wan, Yu Zhang, Tao Zhou, Hong Huo, Tao Fang, "Exploiting Convolutional Neural Networks with Deeply Local Description for Remote Sensing Image Classification", IEEE Access vol. 6, DOI: 10.1109/ACCESS.2018.2798799 , pp. 11215 11228, January 2018.

[7] Pietro Nardelli, Daniel Jimenez-Carretero, David Bermejo-Pelaez, George R. Washko, Farbod N. Rahaghi,Maria J. Ledesma-Carbayo, Ra'ul San Jos'e Est'epar, "Pulmonary Artery-Vein Classification in CT Images Using Deep Learning", IEEE Transactions on Medical Imaging, Vol. 37 ,issue 11, DOI: 10.1109/TMI.2018.2833385, pp. 2428 - 2440, Nov 2018.

[8] Yian Seo, Kyung-shik Shin, "Image classification of fine-grained fashion image based on style using pre-trained convolutional neural network", 2018 IEEE 3rd International Conference on Big Data Analysis (ICBDA), DOI: 10.1109/ICBDA.2018.8367713, March 2018.

[9] Er-Yang Huan, Gui-Hua Wen, Shi-Jun Zhang, Dan-Yang Li, Yang $\mathrm{Hu}$, Tian Yuan Chang, Qing Wang, Bing-Lin Huang, "Deep Convolutional Neural Networks for Classifying Body Constitution Based on Face Image", Computational and Mathematical Methods in Medicine, Volume 2017, Article ID 9846707, Oct 2017.

[10] Tianmei Guo, Jiwen Dong, Henjian Li , Yunxing Gao "Simple Convolutional Neural Network on Image Classification" , 2017 IEEE 2nd International Conference on Big Data Analysis (ICBDA), DOI: 10.1109/ICBDA.2017.8078730, October 2017.

[11] Seong-Hyeon Han, Kwang-Yeob Lee , “Implementation of Image Classification CNN using Mutli thread GPU”, 2017 International SoC Design Conference (ISOCC), DOI: 10.1109/ISOCC.2017.8368904,May 2018.

[12] An Tien Vo ,Hai Son Tran, Thai Hoang Le, “Advertisement Image Classification Using Convolutional Neural Network", 2017 9th International Conference on Knowledge and Systems Engineering (KSE) , DOI: 10.1109/KSE.2017.8119458,pp. 197-202, November 2017.

[13] Ahmed Ali Mohammed Al-Saffar, Hai Tao, Mohammed Ahmed Talab, "Review of Deep Convolution Neural Network in Image Classification", 2017 International Conference on Radar, Antenna, Microwave, Electronics, and Telecommunications, DOI: 10.1109/ICRAMET.2017.8253139,pp. 26-31, Oct 2017.

[14] Ling Zhang , Le Lu , Isabella Nogues , Ronald M. Summers , Shaoxiong Liu , Jianhua Yao, "DeepPap: Deep Convolutional Networks for Cervical Cell Classification", IEEE Journal of Biomedical and Health Informatics ,Vol. 21,issue 6, DOI: 10.1109/JBHI.2017.2705583 pp. 1633 - 1643, Nov 2017.
[15] Hoo-Chang Shin, Holger R. Roth, Mingchen Gao, Le Lu ; Ziyue $\mathrm{Xu}$, Isabella Nogues, Jianhua Yao, Daniel Mollura, Ronald M. Summers "Deep Convolutional Neural Networks for ComputerAided Detection: CNN Architectures, Dataset Characteristics and Transfer Learning", Vol. 35, issue 05, DOI: 10.1109/TMI.2016.2528162, pp. 1285 - 1298,Feburary 2016.

[16] Xuesong Wang, Chen Chen, Yuhu Cheng ,Z. Jane Wang, "Zeroshot Image Classification Based on Deep Feature Extraction", IEEE Transactions on Cognitive and Developmental Systems, Vol 10 ,issue 02, DOI: 10.1109/TCDS.2016.2632178 , pp. 432 - 444, November 2016.

[17] Hicham Riri, Abdelmajid Elmoutaouakkil , Abderrahim BeniHssane , Farid Bourezgui "Classification and recognition of dental images using a decisional tree", 2016 13th International Conference on Computer Graphics, Imaging and Visualization (CGiV), DOI: 10.1109/CGiV.2016.82, pp. 390-393, May 2016.

[18] Holger R. Roth, Christopher T. Lee, Hoo-Chang Shin, Ari Seff, Lauren Kim, Jianhua Yao, Le Lu, Ronald M. Summers "AnatomySpecific Classification Of Medical Images Using Deep Convolutional Nets", 2015 IEEE International Symposium on Biomedical Imaging, DOI: 10.1109/ISBI.2015.7163826,pp. 101 104,April 2015.

[19] Yim, J., Ju, J., Jung, H., \& Kim, J. "Image Classification Using Convolutional Neural Networks With Multi-stage Feature",Robot Intelligence Technology and Applications 3, doi:10.1007/978-3-31916841-8_52,pp. 587-594, 2015.

[20] Olga Russakovsky, Jia Deng, Hao Su1, Jonathan Krause1, Sanjeev Satheesh, Sean Ma,Zhiheng Huang,Andrej Karpathy, Aditya Khosla, Michael Bernstein, Alexander C. Berg,Li Fei-Fei, "Imagenet large scale visual recognition challenge" International Journal of Computer Vision, DOI 10.1007/s11263-015-0816-y, pp. 211-252 ,March 2015.

[21] C. Szegedy, W. Liu, Y. Jia, P. Sermanet, S. Reed, D. Anguelov, D. Erhan, and A. Rabinovich, "Going deeper with convolutions," IEEE Conf. on CVPR, 2015.

[22] Kaiming He, Xiangyu Zhang, Shaoqing Ren, and Jian Sun, "Spatial pyramid pooling in deep convolutional networks for visual recognition". Computer VisionECCV 2014. New York: Springer International Publishing, DOI: arXiv:1406.4729v4, pp. 346361,April 2015.

[23] M. Lin, Q. Chen, S. Yan. "Network in network" [EB / OL]. Http: // arxiv. Org / abs / 1312. 4400,2013.

[24] Krizhevsky A, Sutskever I, Hinton G E. "ImageNet Classification with Deep Convolutional Neural Networks", Advances in Neural Information Processing Systems, 2012, 25(2):2012.

[25] T. Ahonen, A. Hadid, M. Pietikainen, "Face description with local binary patterns: Application to face recognition", IEEE Transactions On Pattern Analysis And Machine Intelligence, Vol. 28,issue 12, pp. 2037-2041,December 2006.

[26] Ajay Khunteta, Preeti Sharma, Sunil Pathak, Ajit Noonia, "Image Security Using Triple Key Chaotic Encryption and SPIHT Compression", International Conference on Artificial Intelligence: Advances and Applications 2019: Proceedings of ICAIAA 2019, Published by Springer Nature, pp 227, 2020. 


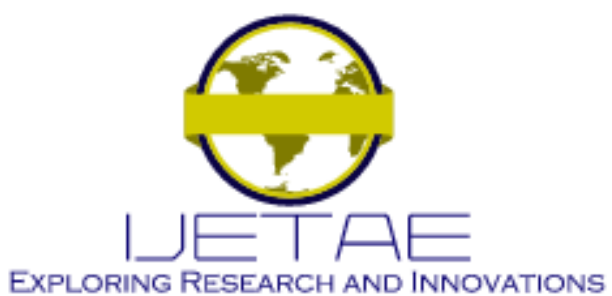

International Journal of Emerging Technology and Advanced Engineering

Website: www.ijetae.com (E-ISSN 2250-2459, Scopus Indexed, ISO 9001:2008 Certified Journal, Volume 12, Issue 01, January 2022)

[27] Jiang, Qingsheng, Dapeng Tan, Yanbiao Li, Shiming Ji, Chaopeng Cai, and Qiming Zheng, "Object detection and classification of metal polishing shaft surface defects based on convolutional neural network deep learning", Applied Sciences, vol. 10, no. 1, pp. 87, 2020.

[28] Liu, Yizhi, Xiaoyan Gu, Lei Huang, Junlin Ouyang, Miao Liao, and Liangran Wu, "Analyzing periodicity and saliency for adult video detection", Multimedia Tools and Applications, vol.79, no. 7, pp. 4729-4745, 2020.

[29] Moreira, Danilo Coura, and Joseana Macêdo Fechine, "A machine learning-based forensic discriminator of pornographic and bikini images", In proceedings of International Joint Conference on Neural Networks (IJCNN), IEEE, pp. 1-8, 2018.

[30] Pierezan, Juliano, and Leandro Dos Santos Coelho, "Coyote optimization algorithm: a new metaheuristic for global optimization problems", In proceedings of IEEE congress on evolutionary computation (CEC), IEEE, pp. 1-8, 2018.

[31] Binu D; Kariyappa B.S, "Rider Deep LSTM Network for Hybrid Distance Score-based Fault Prediction in Analog Circuits", IEEE Transactions on Industrial Electronics, pp.1, October 2020.
[32] Chahi, A., Y. Ruichek, and R. Touahni, "Local directional ternary pattern: A new texture descriptor for texture classification", Computer vision and image understanding, vol. 169, pp. 14-27, 2018.

[33] Kumar, Vijay, and Avneet Kaur, "Binary spotted hyena optimizer and its application to feature selection", Journal of Ambient Intelligence and Humanized Computing, vol. 11, no. 7, pp. 2625 2645, 2020.

[34] Abualigah, Laith, Dalia Yousri, Mohamed Abd Elaziz, Ahmed A. Ewees, Mohammed AA Al-qaness, and Amir H. Gandomi, "Aquila Optimizer: A novel meta-heuristic optimization Algorithm", Computers \& Industrial Engineering, vol. 157, pp. 107250, 2021.

[35] Brown, D.A., I. Craw and J. Lewthwaite," A SOM based approach to skin detection with application in real time systems", Proceeding of the British Machine Vision Conference, (BMVC'01), University of Leeds, UK., pp: 491-500, 2001.

[36] Choudhary, D., Malasri, S.,"Machine learning techniques for estimating amount of coolant required in shipping of temperature sensitive products",(2020) International Journal of Emerging Technology and Advanced Engineering, 10 (10), pp. 67-70. DOI: 10.46338/ijetae1020_12

[37] Al-Thani, M.G., Yang, D., "Machine Learning for the Prediction of Returned Checks Closing Status", (2021) International Journal of Emerging Technology and Advanced Engineering, 11 (6), pp. 19-26. 